\title{
Die Angst vor der Leere
}

\section{Michel Matter}

Dr. med., Vizepräsident der FMH, Departement Dienstleistungen und Berufsentwicklung

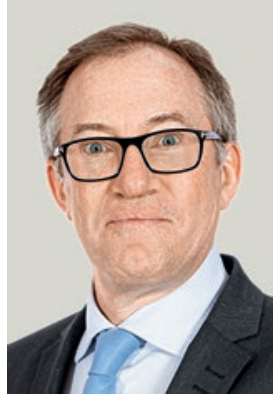

Unser gemeinsamer Weg durch die gesundheitliche, soziale und wirtschaftliche Krise ist lang und beschwerlich. Es besteht aber die Hoffnung, dass die Impfung der vulnerabelsten und schwächsten Mitglieder unserer Gesellschaft schliesslich Licht am Ende des Tunnels bringen wird. Die Entwicklung der letzten Wochen und die entsprechenden Massnahmen des Bundes zeigen, dass der Frühling und womöglich auch der Sommer schwierig werden dürften. Davon sind alle in ihrem beruflichen und privaten Alltag betroffen.

An den Hochschulen sind die Studierenden verzweifelt: kaum noch Kontakt zu Kommilitonen, keine direkte Interaktion mit ihren Professorinnen und insbesondere im Medizinstudium weniger klinische Praxis. Die Pandemie hat alles durcheinandergebracht, was das Risiko einer eingeschränkteren, qualitativ schlechteren Ausbildung birgt. Es gibt nun mal keinen Ersatz für gemeinsames Lernen und Praxiserfahrung, die den Erwerb klinischer Kenntnisse ermöglicht und das Herz unserer medizinischen Ausbildung bildet. Der Übergang von Theorie zu Praxis, vom Online-Dokument zur Realität des Abtastens und Abhörens, ist ein Höhepunkt des Medizinstudiums, wo der weisse Kittel und Beruf zur konkreten Realität werden.

Es gibt nun mal keinen Ersatz für den Übergang von Theorie zu Praxis, vom Online-Dokument zur Realität des Abtastens und Abhörens.

Was die Studierenden der höheren Semester anbelangt, sind sie oft bereits auf Stationen im Einsatz, wo jetzt alles schneller gehen muss, wo der Stress spürbar ist und die Erschöpfung droht. Die Einarbeitung geschieht schnell, und manche finden sich in einer neuen Umgebung wieder, ohne dass ihnen jemand eine Orientierungshilfe gäbe. Rasch müssen sie Verantwortung übernehmen, und für die Einarbeitung auf den Stationen bleibt nur wenig Zeit, da von Assistenzärzten bis hin zu Klinikleiterinnen alle unter Zeitdruck stehen und überarbeitet sind. In diesen Situationen nimmt die Verantwortung gegenüber der Gesellschaft als Ganzes und der Gemeinschaft vor Ort Form an. Der Einsatz der Studierenden unter erschwerten Bedingungen ist eine wertvolle Hilfe bei der Behandlung der hospitalisierten Patientinnen und Patienten, ob nun mit oder ohne Coronavirus.

\section{Der Einsatz der Studierenden unter erschwerten Bedingungen ist eine wertvolle Hilfe bei der Behandlung der hospitalisierten Patientinnen.}

Die psychologischen Auswirkungen der Krise sind immens. Sie hinterlässt in allen Gesellschaftsschichten verheerende Spuren, gerade auch bei den Studierenden. Die jungen Menschen fühlen sich vergessen und an den Rand gedrängt. Ihre Verzweiflung sollte uns sehr zu denken geben. Die Studierenden fürchten sich vor dem Fehlen einer klaren Perspektive für die nächste Zeit, aber auch vor sozialer Isolation, vor einem Schmalspurstudium und vor Prüfungen, für die sie zu wenig lernen und verinnerlichen konnten. Wir müssen endlich ihr Leid zur Kenntnis nehmen, über das in den Medien, zunehmend aber auch von Ärztinnen und Ärzten in Psychiatrie und Grundversorgung berichtet wird.

Als Therapeuten werden wir tagtäglich mit Patientinnen und Patienten konfrontiert, die ihre Niedergeschlagenheit, ihre Einsamkeit, ihr Unbehagen und ihren Verdruss über diese endlos scheinende Situation schildern. Suchtforschende berichten, dass der Konsum psychoaktiver und illegaler Substanzen in die Höhe schnellt. Auch in Kinderpsychiatrie, Psychiatrie und Erstversorgung schlägt man Alarm. Die Gesundheitskrise hat zu einer wirtschaftlichen und sozialen, aber eben auch psychologischen Krise geführt. Unsere Aufgabe ist es deshalb, Schutz und Beistand zu leisten und zu verdeutlichen, dass die jungen Menschen sich dieser Situation hilflos ausgeliefert fühlen. 